\title{
APLICACIÓN DE ÍNDICES ESPECTRALES PARA IDENTIFICAR NECESIDADES DE FERTILIZACIÓN NITROGENADA EN CAFÉ
}

\author{
José Raúl Rendón Sáenz*, Siavosh Sadeghian Khalajabadi**
}

\begin{abstract}
RENDÓN S., J.R.; SADEGHIAN K., S. Aplicación de índices espectrales para identificar necesidades de fertilización nitrogenada en café. Revista Cenicafé 69(1):7-15. 2018
\end{abstract}

El nitrógeno-N ha sido señalado como el nutriente más limitante para la producción de café en Colombia. En esta investigación se planteó como objetivo evaluar la aplicación de índices espectrales para determinar las necesidades de $\mathrm{N}$ en café (Coffea arabica L.) durante la etapa de producción. El experimento se realizó en la Estación Experimental Paraguaicito (Buenavista, Quindío) donde se aplicaron cinco dosis de N (0, 150, 225, 300 y 375 kg.ha-año ${ }^{-1}$ ), suministradas como urea en los meses de abril y octubre de los años 2013 a 2015 , los tratamientos se asignaron bajo un diseño de bloques completos al azar, con cuatro repeticiones. Antes de aplicar los tratamientos, en cada época se evaluó el índice relativo de clorofila-IRC con el equipo SPAD-502 en los tercios inferior, medio y superior de las plantas; así mismo, se valoró el Índice de Vegetación Diferencial Normalizado-NDVI con el equipo GreenSeeker y se registró la producción anual de café cereza. Las lecturas con el SPAD no permitieron determinar con exactitud la necesidad de realizar ajustes en la fertilización nitrogenada para café, los valores promedio de NDVI se redujeron con el incremento en la edad del cultivo expresando el vigor de las plantas, mientras que la producción acumulada presentó respuesta a las dosis crecientes de $\mathrm{N}$, con una tendencia de tipo lineal $(P<0,0001)$.

Palabras clave: Coffea arabica L., nitrógeno, NDVI, SPAD.

\section{APPLICATION OF SPECTRAL INDICES TO IDENTIFY NITROGEN FERTILIZATION NEEDS IN COFFEE}

Nitrogen-N has been pointed out as the most limiting nutrient for coffee production in Colombia. This research aimed to evaluate the application of spectral indexes to determine the needs of $\mathrm{N}$ in coffee (Coffea arabica L.) during the production stage. The experiment was conducted at the Experimental Farm Paraguaicito (Buenavista, Quindío), where five urea $\mathrm{N}$ doses $(0,150,225,300$ and $375 \mathrm{~kg} \cdot$ ha-1 $\cdot$ year-1) were applied in April and October from 2013 to 2015 , the treatments were assigned under a randomized complete blocks design with four replications. Before administering the treatments, the relative chlorophyll-IRC index was evaluated in each season with the SPAD-502 equipment in the lower, middle and upper thirds of the plants. Likewise, the Normalized Differential Vegetation Index-NDVI was evaluated with the GreenSeeker equipment and the annual coffee berry yield was recorded. The SPAD readings did not allow to accurately determine the need to make adjustments to the nitrogen fertilization of coffee, the average values of NDVI were reduced with the increase in the age of the crop by expressing the vigor of the plants, while the accumulated production responded to the increasing doses of $\mathrm{N}$ with a linear trend $(P<0.0001)$.

Keywords: Coffea arabica L., nitrogen, NDVI, SPAD.

\footnotetext{
*Investigador Científico I. Disciplina de Fitotecnia, Centro Nacional de Investigaciones de Café, Cenicafé. Manizales, Caldas, Colombia.

**Investigador Científico III. Disciplina de Suelos, Centro Nacional de Investigaciones de Café, Cenicafé. Manizales, Caldas, Colombia.
} 
El nitrógeno $(\mathrm{N})$ es el elemento que más se refleja en la composición mineral de las hojas de café con las aplicaciones que se hacen al suelo (7), los contenidos del elemento en este órgano de la planta tienen una alta relación con la producción del cultivo (21), por esta razón el $\mathrm{N}$ es el único elemento que no debe excluirse de los planes de fertilización (15), ya que al suprimirse, la producción de café disminuye en promedio hasta un 19\% $(16,18)$.

Para los suelos de la zona cafetera colombiana, las recomendaciones de nutrición nitrogenada se basan principalmente en los contenidos de materia orgánica del suelo (MO), por ser ésta la principal reserva de $\mathrm{N}$ de la cual, a través de procesos de mineralización, se obtiene parte de los requerimientos para la planta. Cuando los contenidos de (MO) son menores a 8,2\% se clasifican como bajos y muy bajos (17). $\mathrm{Si}$ la $\mathrm{MO}$ en el suelo es menor o igual al $8,0 \%$, para la fertilización del café se sugiere utilizar las máximas dosis de N (300 kg.haaño-1), conforme aumentan los contenidos las dosis se disminuyen; en condiciones donde los contenidos de MO superan el 20,0\% y el proceso de mineralización se reduce por bajas temperaturas, es necesario ajustar las recomendaciones incrementando las dosis (15).

Además de la determinación de los contenidos de nutrientes en el suelo, el análisis foliar constituye una medida del estado nutricional del cultivo, con el cual se logra identificar la necesidad de ajustes en los planes de nutrición. Para determinar el contenido mineral en las hojas de especies vegetales se han utilizado métodos destructivos muy específicos y de alto costo, el método Kjeldahl es la técnica más usada para la determinación cuantitativa del $\mathrm{N}$ total. Este tipo de análisis requiere de equipos especializados y reactivos que implican altos costos para la determinación (22).
Otra alternativa para determinar el contenido foliar de elementos como el $\mathrm{N}$, es a través de métodos indirectos (no destructivos) basados en el uso de técnicas de agricultura de precisión, por medio de sensores portátiles con los que se evalúan índices espectrales. Uno de estos sensores es el SPAD (Soil Plant Analysis Development), utilizado para la medición del índice relativo de clorofila en especies vegetales, el cual se relaciona con los contenidos de $\mathrm{N}$ en las hojas. Los valores se basan en la cantidad de luz transmitida por la hoja en longitudes de onda en los $650 \mathrm{~nm}$ (LED rojo) y $940 \mathrm{~nm}$ (LED infrarrojo) (11); el rango de valores de lectura del SPAD es de 0 a 199, por lo que las unidades SPAD serán siempre las mismas, de acuerdo con el tono verde de las hojas (6).

Para evaluar el estado de vigor de los cultivos, su sanidad y nutrición, se ha utilizado el índice de vegetación diferencial normalizado (NDVI), cuyo valor resulta de medir la reflectancia de la radiación en el espectro del rojo $(650 \pm 10 \mathrm{~nm})$ y el infrarrojo cercano $(770 \pm 15 \mathrm{~nm})(19)$. El sensor GreenSeeker (NTech Industries, INc., Ukiah, CA), presenta rangos de medición del NDVI entre $(-1,0$ y 1,0$)$, siendo $0,7-0,8$ los valores más altos, indicadores de plantas en las mejores condiciones (5); este equipo portátil para la evaluación del vigor de los cultivos puede ser una herramienta de interés para establecer la relación entre el índice y las necesidades de nutrición nitrogenada.

Investigaciones desarrolladas para la evaluación de índices espectrales en café a partir del sensor SPAD, han permitido determinar una alta relación entre las lecturas obtenidas con el equipo y la concentración de $\mathrm{N}$ foliar evaluada en plantas de almácigo, indicando que cada unidad de lectura con el equipo equivale a $0,045 \%$ de nitrógeno 
total en las hojas, con un límite superior de 71 unidades (13). Según Franca et al. (2), las lecturas de SPAD observadas en hojas de café con tonalidades verde-claro registran valores entre 25 y 40 unidades, hojas verdes entre 40 y 55 unidades y con tonalidades de verde oscuro lecturas entre 55 y 70 unidades.

En un estudio desarrollado por Reis et al. (14), la concentración de $\mathrm{N}$ foliar $\mathrm{y}$ las lecturas de SPAD en el cultivo de café mostraron correlaciones significativas $(r=$ $0,94)$ cuando se aplicaron dosis crecientes de $\mathrm{N}$, con un valor mínimo de 43,85 y un máximo de 61,95 unidades SPAD. Otro aspecto importante observado en café es la relación que existe entre las lecturas SPAD, la época y el modo de aplicación del fertilizante (8). Lecturas de SPAD efectuadas en cultivos semestrales de tomate (solanum lycopersicum) y maíz (Zea mayz) evidencian una alta relación entre las lecturas de clorofila relativa y la concentración de $\mathrm{N}$ foliar, con coeficientes de determinación $\left(R^{2}\right)$ de 0,96 y 0,88 , respectivamente $(9 ; 12)$.

Pese a los avances en el uso potencial que exhiben estos sensores para la evaluación de índices espectrales, en la caficultura colombiana son pocas las investigaciones (1, 13) desarrolladas sobre la aplicación de estos índices para evaluar el estado de nutrición del cultivo y para el caso puntual de $\mathrm{N}$ no se cuenta con suficiente información que determine, a través de mediciones indirectas, la necesidad de ajustes en los planes de nutrición.

El presente trabajo tuvo como objetivo evaluar la aplicación de índices espectrales para determinar las necesidades de nutrición nitrogenada en el cultivo de café durante la etapa de producción, con el fin de hacer más eficiente la nutrición del cultivo y el uso de los fertilizantes.

\section{MATERIALES Y MÉTODOS}

El estudio se realizó en la Estación Experimental Paraguaicito, en el municipio de Buenavista, Quindío $\left(75^{\circ} 44^{\prime} \mathrm{W}, 4^{\circ} 23^{\prime} \mathrm{N}\right)$, altitud $1.203 \mathrm{~m}$, en la zona cafetera central de Colombia. Las características de clima y suelo se presentan en la Tabla 1. La investigación se realizó en un lote de café (Coffea arabica) Variedad Castillo $^{\circledR}$, con una densidad de siembra de 6.666 plantas/ha. Se evaluaron cinco tratamientos, que consistieron en dosis crecientes de $\mathrm{N}$, aplicadas luego de la siembra del cultivo.

Tabla 1. Características de clima y suelo en la Estación Experimental Paraguaicito.

\begin{tabular}{|c|c|}
\hline \multicolumn{2}{|c|}{ Variables de clima } \\
\hline Temperatura media $\left({ }^{\circ} \mathrm{C}\right)$ & 22,2 \\
\hline Precipitación (mm.año-1) & $2.179,0$ \\
\hline Brillo solar (h.año-1) & $1.757,0$ \\
\hline Humedad relativa (\%) & 78,5 \\
\hline \multicolumn{2}{|c|}{$\begin{array}{c}\text { Propiedades del suelo } \\
\end{array}$} \\
\hline $\mathrm{pH}$ & 4,60 \\
\hline Materia orgánica (\%) & 7,20 \\
\hline Nitrógeno $(\%)$ & 0,31 \\
\hline Fósforo $\left(\mathrm{mg} \cdot \mathrm{kg}^{-1}\right)$ & 130,00 \\
\hline Potasio $\left(\mathrm{cmol}_{(+)} \cdot \mathrm{kg}^{-1}\right)$ & 0,61 \\
\hline Calcio $\left(\mathrm{cmol}_{(+)} \cdot \mathrm{kg}^{-1}\right)$ & 1,52 \\
\hline Magnesio $\left(\mathrm{cmol}_{(+)} \cdot \mathrm{kg}^{-1}\right)$ & 0,39 \\
\hline Aluminio $\left(\mathrm{cmol}_{(+)} \cdot \mathrm{kg}^{-1}\right)$ & 1,50 \\
\hline
\end{tabular}

Durante la etapa de crecimiento vegetativo del cultivo hasta los 18 meses de edad se aplicaron en total dosis de $\mathrm{N}$ de $(0,0,29,0$, 44,0, 58,0 y 73,0 g/planta), una vez inició la etapa reproductiva se aplicaron dosis de $\mathrm{N}$ de $0,0,150,0,225,0,300,0$ y 375,0 kg.ha-año-1 durante los años 2013, 2014 y 2015, fraccionando las dosis de cada año en dos aplicaciones, realizadas en los meses de abril y octubre, en época de lluvias. Como fuente comercial de nitrógeno se utilizó urea $(46 \% \mathrm{~N})$, los demás nutrientes 
se suministraron según las recomendaciones del análisis de suelo.

Los tratamientos se dispusieron bajo un diseño de bloques completos al azar, con cuatro repeticiones. Cada parcela experimental estuvo constituida por 28 plantas de café, de las cuales se seleccionaron cuatro plantas centrales para realizar las evaluaciones. Como variables de respuesta se registró la producción en gramos de café cereza por planta $\left(\mathrm{kg} \cdot \mathrm{ha}^{-1}\right)$. Antes de la fertilización, en los meses de abril y octubre, se realizaron mediciones del índice relativo de clorofila con el equipo SPAD-502 y el índice normalizado de vegetación (NDVI) con el equipo GreenSeeker. Las lecturas con el equipo SPAD-502 se hicieron dividiendo la planta de café en tres estratos, el estrato superior el cual corresponde a la zona de formación de nuevas ramas primarias, el estrato medio donde se encuentra la zona más productiva del árbol y el estrato inferior conformado por las ramas de mayor edad. En una de las caras del árbol se seleccionó una rama de cada estrato y en cada rama seleccionada se tomó una lectura en una de las hojas del tercer par, contadas desde el ápice, ubicando el equipo en la parte central de la hoja, al lado de la nervadura.
En los mismos árboles donde se realizó la lectura SPAD, también se registró el índice normalizado de vegetación (NDVI) con el equipo GreenSeeker (NTech Industries, INc., Ukiah, CA), desplazando el sensor a una distancia de $50 \mathrm{~cm}$ aproximadamente, en una de las caras del árbol.

Los datos de producción y las lecturas de SPAD y NDVI, fueron analizados a partir del análisis de varianza (ANAVA) y el análisis de regresión, según el diseño experimental aplicado. La prueba de Duncan, al nivel de significancia del 5\%, se realizó para determinar la diferencia entre medias. El análisis estadístico se realizó a través del Software SAS versión 9.4 (SAS Institute, 2012).

\section{RESULTADOS Y DISCUSIÓN}

Índice relativo de clorofila. Los registros del índice relativo de clorofila obtenidos con el equipo SPAD, en los tres estratos de los árboles de café, mostraron para el estrato superior valores significativamente superiores a los obtenidos en los estratos medio e inferior (Figura 1). Las diferencias estadísticas entre los promedios de las unidades SPAD pueden asociarse a la dinámica del crecimiento de

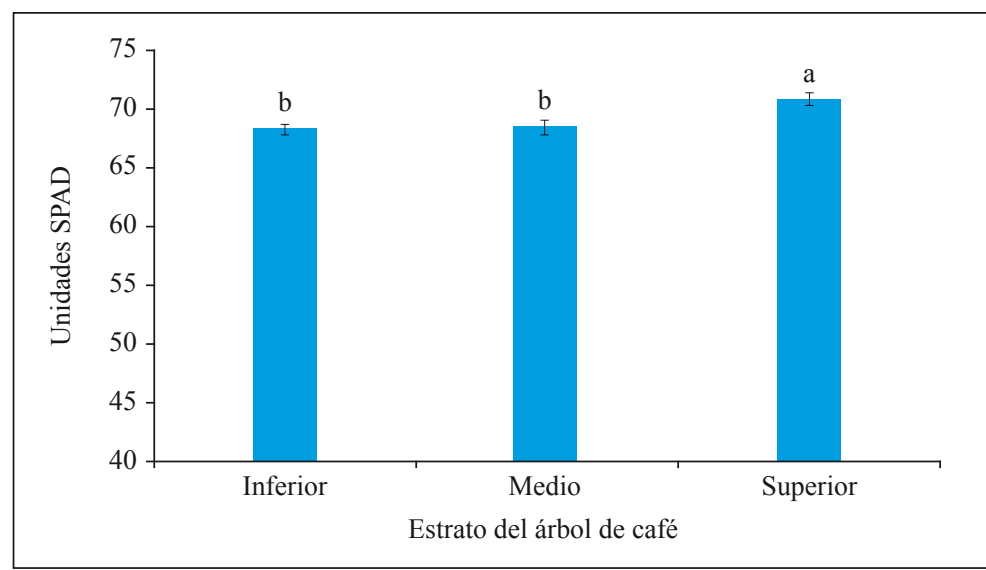

Figura 1. Unidades de lectura SPAD promedio en los estratos inferior, medio y superior del árbol de café y prueba de comparación Duncan $(\operatorname{Pr}<0,05)$. 
las plantas de café y a la movilidad del $\mathrm{N}$. Con relación a la movilidad del $\mathrm{N}$ en las plantas, ésta se caracteriza por presentar la mayor concentración del elemento en las ramas y hojas más jóvenes (10). En los árboles de café todas las ramas pueden formar nuevas hojas, sin embargo, aquellas que se encuentran en la zona productiva tienden a presentar menores contenidos de $\mathrm{N}$, en comparación con las hojas nuevas ubicadas en el estrato superior del árbol, lo que explica que los mayores valores de SPAD se hayan registrado en el estrato superior, donde es menos probable encontrar frutos extrayendo el elemento. Los resultados obtenidos con el SPAD conservan similitud a los reportados por Reis et al. (14) en café variedad Catuaí, con las mayores lecturas de SPAD registradas en la zona apical de la planta.

Considerando el estrato medio como la zona en la cual se concentra la mayor producción de los árboles café, a su vez donde más relación existe entre el $\mathrm{N}$ y la producción (21) y que corresponde al mismo sitio en el cual se toman las muestras para el análisis foliar, se optó por analizar las lecturas SPAD obtenidas en este lugar como un indicador de las necesidades de $\mathrm{N}$ para el cultivo en la etapa de producción.

Los promedios de unidades SPAD obtenidos en el estrato medio del árbol de café, en respuesta a las dosis crecientes de $\mathrm{N}$, se presentan en la Tabla 2. Según el análisis de varianza aplicado para el diseño, se encontraron diferencias significativas sólo entre los promedios obtenidos en la evaluación del segundo semestre de 2014. Los promedios de SPAD registrados en el segundo semestre del año 2013, con un mínimo de 70,6 para el tratamiento $\sin \mathrm{N}$ y un máximo de 78,3 para la dosis de $300 \mathrm{~kg} \cdot$ ha-año ${ }^{-1}$, conservan relación con el menor volumen de cosecha registrado durante este año. En el sitio de estudio se presenta un patrón de distribución anual de la cosecha que corresponde al $60 \%$ en el primer semestre y un $40 \%$ en el segundo semestre. Para el año 2013 la cantidad de café recolectado en el primer semestre fue sólo el $8 \%$ de la cosecha anual y la cantidad cosechada durante el año fue menos del $40 \%$ del total recolectado en los años 2014 y 2015.

Puede considerarse que a partir de las unidades de lectura SPAD obtenidas en este estudio y la escasa relación encontrada con las dosis de $\mathrm{N}$ aplicadas, no es posible determinar con exactitud la necesidad de realizar ajustes en la fertilización nitrogenada de plantaciones de café en etapa de producción. Con base en observaciones similares, Gianquinto et al. (3) expresan limitaciones de las lecturas SPAD, para detectar de manera puntual el estado sub-óptimo del $\mathrm{N}$ en el cultivo de papa (Solanum tuberosum), en respuesta a la fertilización con dosis medias y altas de $\mathrm{N}$, dado que no se encontraron diferencias entre las aplicaciones de dichas dosis.

En contraste, Grava et al. (4) encontraron respuesta a las dosis crecientes de $\mathrm{N}$, al evaluar el índice relativo de clorofila en diferentes estados fenológicos del cultivo de café bajo fertirrigación, con una tendencia de tipo lineal e incrementos de aproximadamente dos unidades en las lecturas de SPAD por cada $100 \mathrm{~kg} \cdot \mathrm{ha}^{-1}$ de $\mathrm{N}$ aplicado.

Índice normalizado de vegetación. Los registros de NDVI obtenidos con el equipo GreenSeeker en las plantas de café, mostraron efecto de los tratamientos según el análisis de varianza en las evaluaciones realizadas durante los años 2014 y 2015 (Figura 2). Durante estos años, el promedio del NDVI registrado para el tratamiento $\sin \mathrm{N}$ fue menor (entre 0,06 y 0,08 unidades) al promedio obtenido con la dosis de $300 \mathrm{~kg}$.ha-año- ${ }^{-1}$. 
Tabla 2. Promedios (Prom.) y error estándar (E.E.) para las unidades SPAD en el estrato medio del árbol de café con las dosis crecientes de $\mathrm{N}$.

\begin{tabular}{|c|c|c|c|c|c|c|c|c|c|c|c|c|}
\hline \multirow{3}{*}{ Año } & \multirow{3}{*}{ Semestre } & \multicolumn{10}{|c|}{ Dosis N (kg.ha-año-1) } & \multirow{3}{*}{$\operatorname{Pr}>\boldsymbol{F}$} \\
\hline & & \multicolumn{2}{|c|}{$\mathbf{0}$} & \multicolumn{2}{|c|}{150} & \multicolumn{2}{|c|}{225} & \multicolumn{2}{|c|}{300} & \multicolumn{2}{|c|}{375} & \\
\hline & & Prom. & E.E. & Prom. & E.E. & Prom. & $\overline{\text { E.E. }}$ & Prom. & $\overline{\text { E.E. }}$ & Prom. & $\overline{\text { E.E. }}$ & \\
\hline \multirow{2}{*}{2013} & 1 & $\overline{59,381}$ & $\overline{5,719}$ & $\overline{60,719}$ & $\overline{6,120}$ & $\overline{60,950}$ & $\overline{2,634}$ & $\overline{62,725}$ & $\overline{3,307}$ & $\overline{65,756}$ & $\overline{1,687}$ & 0,1912 \\
\hline & 2 & 70,594 & 1,819 & 74,506 & 2,971 & 76,525 & 1,068 & 78,319 & 2,473 & 76,956 & 2,116 & 0,2078 \\
\hline \multirow{2}{*}{2014} & 1 & 65,163 & 1,898 & 68,763 & 1,069 & 69,392 & 1,629 & 67,338 & 2,767 & 69,631 & 1,109 & 0,5180 \\
\hline & 2 & 57,288 & 1,899 & 68,606 & 3,477 & 67,213 & 3,237 & 70,244 & 1,787 & 72,456 & 2,165 & 0,0006 \\
\hline \multirow{2}{*}{2015} & 1 & 62,713 & 2,915 & 68,869 & 0,974 & 69,688 & 2,801 & 68,738 & 1,743 & 70,356 & 1,710 & 0,3085 \\
\hline & 2 & 61,275 & 2,687 & 63,594 & 2,172 & 66,650 & 1,286 & 66,256 & 1,447 & 68,275 & 2,102 & 0,2437 \\
\hline
\end{tabular}

En general, para las lecturas de este índice, se observó la disminución de los valores promedio de NDVI con el incremento en la edad del cultivo, esta condición pudo asociarse con la pérdida de ramas bajeras en las plantas, el desplazamiento de la producción hacia la parte superior del árbol y la disminución de la producción en el cultivo cuando se completa el ciclo de producción. Silva et al. (20) en plantaciones de café, encontraron variación de los valores de NDVI en función de la época en la que se realizan las evaluaciones, y mencionan que la pérdida del volumen de hojas en períodos con menor precipitación y cuando disminuyen temperaturas, provocan la reducción del índice NDVI.
El índice de vegetación como herramienta para diagnosticar necesidades extremas de nutrición en plantaciones de café, puede tener un uso potencial en vista de que expresa el estado de vigor de las plantas en sus etapas de desarrollo; sin embargo, presenta limitaciones para identificar las necesidades puntuales relacionadas con la nutrición nitrogenada en el cultivo de café.

Producción de café cereza. Al analizar la producción de café cereza registrada por cada año, se detectó un aumento relativo de esta variable con las dosis crecientes de $\mathrm{N}$ aplicadas, mostrando efecto sobre la producción obtenida durante el año 2015

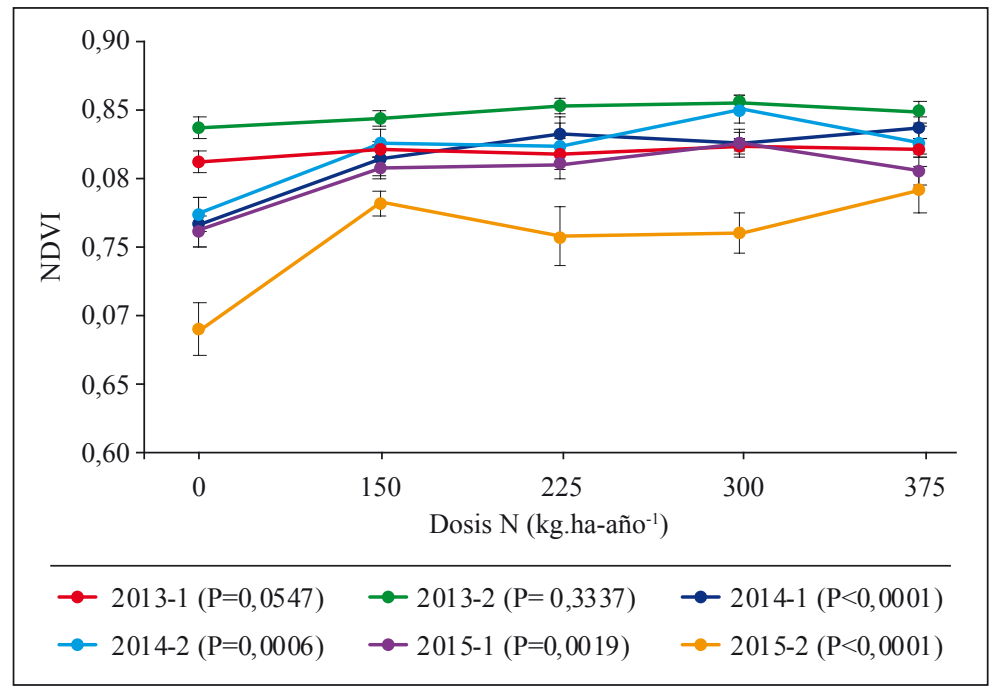

Figura 2. Lecturas promedio del Índice de Vegetación Diferencial Normalizado -NDVI en función de las dosis crecientes de $\mathrm{N}$ y la edad del cultivo. 
según el análisis de varianza $(P=0,0418)$. A partir de estos resultados, con una dosis de 300 kg.ha-año ${ }^{-1}$ de $\mathrm{N}$, el incremento en la producción fue del $33,6 \%$ en 2013 , del $23,9 \%$ en 2014 y del $43,0 \%$ en 2015 en comparación con el tratamiento donde no se aplicó N (Tabla 3).

La producción acumulada de café cereza por hectárea en los tres años evaluados, mostró efecto de las dosis de $\mathrm{N}$ según el análisis de varianza $(P=0,0018)$. Para esta misma variable se determinó que la respuesta a la fertilización nitrogenada con dosis crecientes se ajustó a una tendencia de tipo lineal $(P$ $<0,0001)$, con un coeficiente de determinación $R^{2}=0,96$ (Figura 3).

El efecto de las dosis crecientes de $\mathrm{N}$ sobre la producción acumulada de café cereza permitió determinar el mayor incremento en la producción $(33,9 \%)$, cuando se comparó la

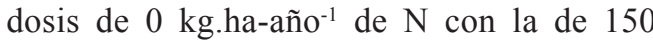
$\mathrm{kg}$.ha-año $0^{-1}$ y un menor incremento sobre la producción $(4,0 \%)$ cuando se aumentó la dosis de $\mathrm{N}$ de 300 a 375 kg.ha-año-1 (Figura 2).

Los resultados de este estudio permiten concluir que a partir del índice relativo de clorofila obtenido con el equipo SPAD, las evaluaciones realizadas para el diagnóstico del estado nutricional del cultivo en etapa de producción no reflejan las necesidades de ajuste en los planes de nutrición nitrogenada. El índice normalizado de vegetación obtenido con el equipo GreenSeeker, permite diagnosticar necesidades extremas de nutrición en plantaciones de café, pero presenta limitaciones para identificar las necesidades puntuales relacionadas con la nutrición nitrogenada en el cultivo de café.

Tabla 3. Promedios (Prom.) y error estándar (E.E.) para la producción anual de café cereza en $\mathrm{kg}$.ha ${ }^{-1}$ según las dosis crecientes de $\mathrm{N}$.

\begin{tabular}{|c|c|c|c|c|c|c|c|c|c|c|}
\hline \multirow{3}{*}{ Año } & \multicolumn{10}{|c|}{ Dosis N (kg.ha-año-1) } \\
\hline & \multicolumn{2}{|c|}{0} & \multicolumn{2}{|c|}{150} & \multicolumn{2}{|c|}{225} & \multicolumn{2}{|c|}{300} & \multicolumn{2}{|c|}{375} \\
\hline & Prom. & E.E. & Prom. & E.E. & Prom. & E.E. & Prom. & E.E. & Prom. & E.E. \\
\hline 2013 & 6.752 & 2.301 & 9.479 & 4.868 & 8.247 & 847 & 10.174 & 1.628 & 9.960 & 2.200 \\
\hline 2014 & 18.579 & 3.129 & 20.365 & 1.760 & 21.823 & 1.918 & 24.416 & 2.976 & 24.648 & 3.491 \\
\hline 2015 & 19.128 & 2.049 & 29.693 & 1.585 & 33.636 & 29.35 & 33.548 & 1.263 & 36.308 & 3.404 \\
\hline
\end{tabular}

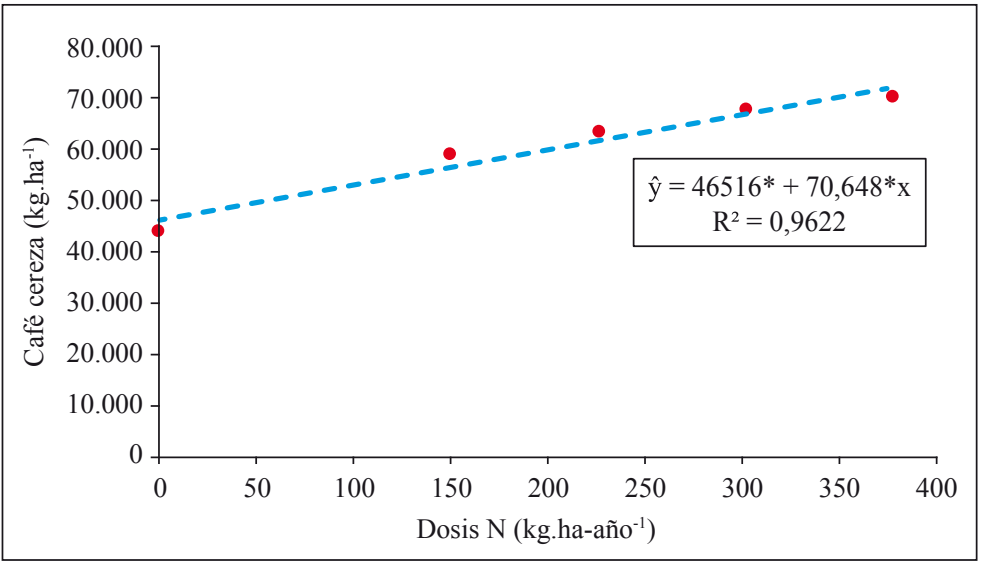

Figura 3. Producción acumulada de café cereza en función de las dosis crecientes de N. 
Para las condiciones del sitio evaluado, la aplicación de $\mathrm{N}$ con dosis superiores a los 300 kg.ha-año ${ }^{-1}$ mostró bajos incrementos en la producción acumulada de café cereza.

\section{AGRADECIMIENTOS}

Se agradece a todas las personas que participaron en las evaluaciones y el manejo agronómico del experimento, a Carlos Augusto Ramírez (Auxiliar de investigación de la Disciplina de Fitotecnia), a Myriam Cañón (Coordinadora de la Estación Experimental Paraguaicito) y Daniel Franco (Auxiliar de investigación de la Disciplina de Experimentación).

\section{LITERATURA CITADA}

1. DÍAZ, M.C. Evaluación del dispositivo portátil SPAD-502 como indicador de la concentración de nitrógeno en plantas de café" coffea Arabica. Pereira : Universidad Tecnológica de Pereira. Facultad de Ciencias Ambientales, 2014. 103 p. Tesis: Maestría en ecotecnología.

2. FRANCA, A.C.; SOUZA, I.F.D.; ALVES, L.W.R.; JESUS, A.M.S. Calibração do medidor de clorofila minolta spad-502 para avaliação do conteúdo de clorofila em cafeeiro. Brasília : Embrapa café, 2007.

3. GIANQUINTO, G.; GOFFARTE, J.E.; OLIVER, M.; GUARDA G.; COLAUZZP, M.; COSTA D., L.; DELLEVEDOVE. G.; VOS, J.; MACKERRON, D.K.L. Chlorophyll meters as a tool to assess the nitrogen status and to guide nitrogen fertilization of potato crop. Potato research 47(1/2):35-80. 2004.

4. GRAVA DE G., L.J.; SANTOS, T.; VILLAS B., R.L.; LEITE J., J.B. Índice relativo de clorofila e o estado nutricional em nitrogênio durante o ciclo do cafeeiro fertirrigado. Revista brasileira de ciência do solo 32(1):217-226. 2008.

5. GUTIÉRREZ S., M.V.; CADET P., E.; RODRÍGUEZ M., W.; ARAYA A, J.M. GreenSeekerTM y el diagnóstico del estado de salud de los cultivos. Agronomía mesoamericana 22(2):397-403. 2011.
6. KRUGHB.,L.B.; MILES, D. The solid-state chlorophyll meter, a novel instrument for rapidly and accurately determining the chlorophyll concentrations in seedling leaves: Maize genetics cooperation. Newsletter 68:25-27. 1994.

7. MALAVOLTA, E. Fertilización foliar. p. 305-341. En: SILVA M., F. Fertilidad de suelos, diagnóstico y control. Bogotá : SCCS, 2001. 507 p.

8. MARTINS, L.E.C.; FURLANI, E.; SANTOS, D.M.A.D.; FERRARI, S.; VAL, H.C.D. Avaliação de leituras spad de acordo com o modo de aplicação de nitrogênio em cafeeiro. Brasília : Embrapa Café, 2007.

9. MENDOZA, M.; GONZÁLEZ, G.A.; SANTELISES, A.A.; ETCHEVERES, J.D.; RINCÓN, J.A. Estimación de la concentración de nitrógeno y clorofila en tomate mediante un medidor portátil de clorofila. Terra 16(2):135-141. 1998.

10. MENGEL, K.; KIRKBY, E.A. Principles of plant nutrition. 4a. ed. Bern: International potash institute, 1978.687 p.

11. MINOLTA CAMERA. Chlorophyll meter SPAD-502: Instruction manual. Osaka : Minolta, 1989. 22 p.

12. NOVOAS., R.; VILLAGRÁNA., N. Evaluación de un instrumento medidor de clorofila en la determinación de niveles de nitrógeno foliar en maíz. Agricultura técnica 62(1):166-171. 2002.

13. RAMÍREZ B., V.H.; MORENO B., A.M.; LÓPEZ R., J.C. Evaluación temprana de la deficiencia del nitrógeno en café y aplicaciones. Manizales : Cenicafé, 2012. 8 p. (Avances Técnicos No. 420).

14. REIS, A.R.; FURLANI J., E.; BUZETTI, S.; ANDREOTTI, M. Diagnóstico da exigência do cafeeiro em nitrogênio pela utilização do medidor portátil de clorofila. Bragantia 65(1):163-171.2006.

15. SADEGHIAN K., S. Guía práctica fertilidad del suelo y nutrición del café en Colombia. Manizales : Cenicafé, 2008. 43 p. (Boletín Técnico No. 32).

16. SADEGHIAN K., S. Efecto de la fertilización con nitrógeno, fósforo, potasio y magnesio sobre las propiedades químicas de suelos cultivados en café. Cenicafé 54(3):242-257. 2003.

17. SADEGHIAN K, S. Respuesta de cafetales al sol y bajo semisombra a nitrógeno y su relación con la materia orgánica del suelo. Facultad nacional de agronomía Medellín 64(1):5781-5791. 2011. 
18. SADEGHIAN K., S.; GARCÍA L., J.C.; MONTOYA R., E.C. Respuesta del cafeto a la fertilización con $\mathrm{N}, \mathrm{P}, \mathrm{K}$ y $\mathrm{Mg}$ en dos fincas del departamento del Quindío. Cenicafé 57(1):58-69. 2006.

19. SAMBORSKI, S.M.; TREMBLAY, N.; FALLON, E. Strategies to make use of plant sensors-based diagnosticinformation fornitrogenrecommendations. Agronomy journal 101(4):800-816. 2009.

20. SILVA, L.C.; SILVA F., M.P.; AGUIAR, F.A.; NAVES, V.L.; LIRAS., J.M.; RAMBAL, S.; MOUILLOT, F.; RODRIGUES, J.P.; BARBOSA, A.D. Uso do índice de vegetação por diferença normalizada (NDVI) na avaliação do desenvolvimento de cafeeiros do sul de Minas Gerais. Curitiba : Simpósio de pesquisa dos cafés do Brasil, 2015.

21. VALENCIA A., G.E.; ARCILA P., J. Efecto de la fertilización con N, P, K, a tres niveles de la composición mineral de las hojas del cafeto. Cenicafé 28(4):119-138. 1977.

22. WOOD, C.W.; REEVES, D.W.; HIMELRICH, D.G. Relationships between chlorophyll meter reading and crop chlorophyll concentration, $\mathrm{N}$ status, and yield: A review. Proceedings agronomy society of New Zealand 23:1-9. 1994. 